Europhysics Letters

PREPRINT

\title{
Universal Fluctuations of the Danube Water Level: a Link with Turbulence, Criticality and Company Growth
}

\author{
Steven.T. Bramwell ${ }^{1}\left({ }^{*}\right)$, Tom Fennell ${ }^{2}$, Peter C. W. Holdsworth $\left.{ }^{3}{ }^{* *}\right)$ and \\ BAPTISTE PORTELLI ${ }^{3}$ \\ 1 University College London, Department of Chemistry, 20 Gordon Street, London \\ WC1H OAJ, UK. \\ 2 The Royal Institution of Great Britain, 21 Albemarle Street, London W1X 4BS, UK. \\ 3 Laboratoire de Physique, ENS-Lyon and CNRS, 46 Allée d'Italie, 69007 Lyon, France.
}

\begin{abstract}
PACS. 2.50 r - Probability theory, stochstic processes, statistics.
PACS. 5.40 -a - Fluctuation phenomena, random processes, noise, Brownian motion.

PACS. 5.65+b - Self-organized systems.
\end{abstract}

\begin{abstract}
A global quantity, regardless of its precise nature, will often fluctuate according to a Gaussian limit distribution. However, in highly correlated systems, other limit distributions are possible. We have previously calculated one such distribution and have argued that this function should apply specifically, and in many instances, to global quantities that define a steady state [1]. Here we demonstrate, for the first time, the relevance of this prediction to natural phenomena. The river level fluctuations of the Danube [2] are observed to obey our prediction, which immediately establishes a generic statistical connection between turbulence, criticality and company growth statistics.
\end{abstract}

Jánosi and Gallas have analysed statistics of the daily water level fluctuations of the Danube collected over the major part of the $20^{\text {th }}$ century [2]. In Figure 1 we show their histogram $P(h)$ of seasonally adjusted river height fluctuations. The data are plotted in the form $\sigma P$ against $\left(\frac{h-\bar{h}}{\sigma_{h}}\right)$, where $\bar{h}$ and $\sigma_{h}^{2}$ are respectively the seasonal mean and varience. This is the form that allows direct comparison of experiment with limit functions such as the normal distribution. The data are compared with one such limit function, the universal probability density function $f(x)$ that gives exactly the thermodynamic limit distribution of two model quantities - (a) the critical order parameter fluctuations of the 2D XY model of magnetism and (b) the steady state width fluctuations of the 2D Edwards-Wilkinson model of interface growth [3]. In the Figure, $f(x)$ was obtained by fast Fourier transform of the exactly solved characteristic function [3]. An excellent comparison is obtained even though Fig.1. contains no fitting parameters. As discussed in more detail below, $f(x)$ is asymmetric, with the tail for fluctuations above the mean going as $x \exp (-x)$, allowing a probability for a large positive fluctuation that is much larger than that predicted by a normal distribution. For example, the largest fluctuation of 5 meters, about $5.5 \sigma_{h}$, during the 87 years of data

(*) E-mail: s.t.bramwell@ucl.ac.uk

$\left({ }^{* *}\right)$ E-mail: peter.holdsworth@ens-lyon.fr

(C) EDP Sciences 


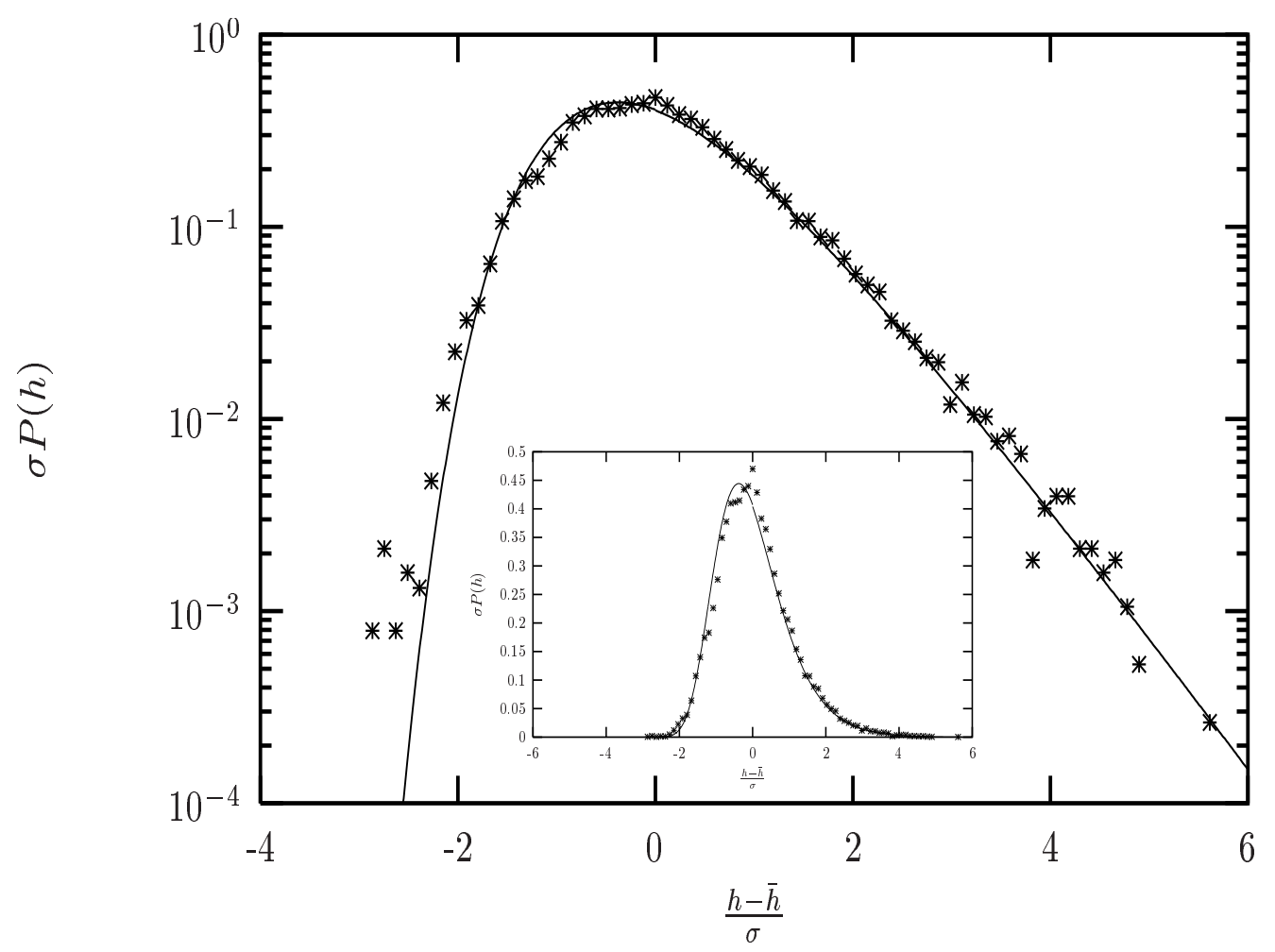

Fig. 1 - Comparison, with no fitting parameters, between (symbols) the histogram $P(h)$ of the seasonally adjusted water level fluctuations of the Danube [2] and (line) the predicted function for complex systems $f(x)$ [3]. The data are plotted as $\sigma P$ against $\left(\frac{h-\bar{h}}{\sigma_{h}}\right)$, where $\bar{h}$ and $\sigma^{2}$ are respectively the seasonal mean and variance. For the Danube data $\sigma_{h}=83 \mathrm{~cm}$, so the fluctuations go out to nearly 5 metres to the high water side. Inset: same as above, in natural units.

collection, is a once per century event for $f(x)$ while a Gaussian probability predicts that such an event only occurs once every 25 millenia. Fluctuations below the mean are of much smaller amplitude, with the tail of the distribution going as $\exp (-\exp (x))$. On the semi-log plot, which emphasises the tails of the distribution, the experimental data coincide with the theoretical function to an excellent degree. In natural units (see inset) the two show some deviation near the origin but again close agreement in the tails.

The striking result shown in Fig.1 gives weight to our prediction that $f(x)$ should occur widely in nature. Specifically, we have predicted that, if the steady state of a complex system is defined by a global or spatially averaged quantity $x$, then the latter will, in many cases, fluctuate according to $f(x)$. This prediction was motivated by the observation that $f(x)$ appears to describe fluctuations of an experimental quantity that is seemingly unrelated to either magnetism or interface growth: the global power consumption of a turbulent flow in nonequilibrium steady state [1]. Subsequently, $f(x)$ was observed in a range of numerical models of self-organised criticality, equilibrium critical behaviour and percolation phenomena 《t]. With the exception of turbulence, these examples are all theoretical. However, the Danube data now provides a radically different experimental system showing this universal behaviour. We observe that the close comparison between theory and experiment in Fig. 1 is obtained 
without any information of the actual mechanism of water level fluctuations. In general water level depends on many complex interacting factors, both naturally occurring and under human control. These include precipitation, evaporation, runoff, snowmelt and percolation [5]. Such factors must ultimately determine the shape of the experimental curve in Fig. 1, but the comparison with $f(x)$ supports the validity of an alternative phenomenological description, in which the Danube water level is regarded as a direct and global measure of water transfer over the river basin.

The data collapse shown in Fig.1 also supports a conjecture made by Jánosi and Gallas [2] that was motivated by work of Stanley et al on the statistics of company growth [6]. Jánosi and Gallas showed that the time series for the Danube data and that for company growth data share common statistical properties. They therefore proposed that these are representative of a wider class of complex systems [2]. Figure 1 indeed suggests a generic connection with turbulence, critical phenomena and interface growth.

Returning now to the details of the data collapse: a limit distribution for a global quantity $y$ is calculated by considering the distribution of $x=\left(\frac{y-\bar{y}}{\sigma_{y}}\right)$ in the limit of infinite system size (the thermodynamic limit). Here $\bar{y}$ is the mean and $\sigma_{y}$ the standard deviation. In an uncorrelated or weakly correlated system, the central limit theorem applies, and the quantity $x$ will obey a normal or Gaussian distribution (an example is the total energy of an ideal gas in the canonical ensemble [7]). However, in a strongly correlated system, the central limit theorem can break down, which means that the limit function can be non-Gaussian. We define $f(x)$ as the non-Gaussian thermodynamic limit distribution that has been shown to exactly describe the two model quantities (a) and (b) listed above [3]. The probability density function $f(x)$ can be obtained by numerical Fourier transform of its exactly known characteristic function $\varphi(t)$. We present here a straightforward derivation of $\varphi(t)$ which is useful to illustrate the relationship of $f(x)$ with other standard distributions.

Consider a global quantity $S=\sum_{\mathbf{n}} s_{\mathbf{n}}$, where $\mathbf{n}$ is a $d$-dimensional vector of integer elements $\pm 1 \ldots \infty$ and each $s_{\mathbf{n}}$ is a gamma variable with probability density function

$$
g\left(s_{\mathbf{n}}\right)=\frac{a_{\mathbf{n}}^{\gamma}}{\Gamma(\gamma)} e^{-a_{\mathbf{n}} s_{\mathbf{n}}} s_{\mathbf{n}}^{\gamma-1}
$$

Here $\gamma=\frac{1}{2}, a_{\mathbf{n}}=|n|^{m}$ and $m$ is a positive integer, specified below.

The logarithm of the characteristic function $\psi_{\mathbf{n}}(t)$ of $s_{\mathbf{n}}$ may be written as an expansion in cumulents $\kappa_{r}\left(s_{\mathbf{n}}\right)$ :

$$
\log \psi_{\mathbf{n}}(t)=\sum_{1}^{\infty} \frac{(i t)^{r}}{r !} \kappa_{r}\left(s_{\mathbf{n}}\right)
$$

where the $r$ th cumulent, $\kappa_{r}(s)=\gamma(r-1) ! a_{\mathbf{n}}^{-r}$ [8]. Using the property that the gamma variables are statistically independent, the characteristic function of the compound variate $S$ is the product of the contributions for each $\mathbf{n}$ : $\Psi(t)=\prod_{\mathbf{n}} \psi_{\mathbf{n}}(t)$, so the $r$ th cumulent of $S$ is simply the sum of the $\kappa_{r}\left(s_{\mathbf{n}}\right)$ :

$$
\kappa_{r}(S)=\frac{1}{2}(r-1) ! \sum_{\mathbf{n}}\left(\frac{1}{|n|^{m}}\right)^{r} .
$$

The limit function is obtained by normalising the variate $S$ by its standard deviation $\sigma_{S}$. The 
$r$ th cumulent of $x=S / \sigma_{S}$ in the expansion of the characteristic function $\varphi(t)$ is:

$$
\kappa_{r}(x)=\frac{\frac{1}{2}(r-1) ! \sum_{\mathbf{n}}\left(\frac{1}{|n|}\right)^{r m}}{\left(\frac{1}{2} \sum_{\mathbf{n}}\left(\frac{1}{|n|}\right)^{2 m}\right)^{r / 2}} .
$$

The second, third and fourth cumulents are respectively the variance, skewness and defect of kurtosis of $x$, and the full probability density function of $x$ can be found by inverse Fourier transform of its characteristic function. For general $m$ and $d$ the shape of the probability density function depends on the relative importance of the small $|n|$ and large $|n|$ contributions. For small $d$ and large $m$ only the $|n|=1$ contribution is important. There are only $2 d$ contributions for $|n|=1$ and the function therefore tends to a $\chi^{2}$ distribution for $2 d$ degrees of freedom. For large $d$ and small $m$ the function tends to a normal distribution as many equivalent large $|n|$ contributions dominate the sum and the second cumulent becomes large compared to all the others (which is essentially the scenario in the central limit theorem).

In the magnetic model discussed in the abstract, the global quantity, the magnetization per spin, is the sum of contributions from $N$ highly correlated spins, but this can be re-cast as contributions from $N$ independent spin wave modes. The probability density may be derived in the low temperature approximation, where each mode gives a statistically independent contribution to the global quantity of the form (11), but with $a=(J / T)|q|^{m}$ where $J$ is the coupling, $T$ the temperature, $m=2$ and $q$ is the wavevector defined in a two-dimensional reciprocal space. Normalization with respect to the standard deviation removes all dependence on coupling and system size from the problem and results, after taking the thermodynamic limit, in the function (4) for the case $d=m=2$. A detailed study of this function gives the asymptotes described above [3]. For $d=m=2$, the shape of $f(x)$ reflects the importance of both small $|n|$ and large $|n|$ contributions, which correspond to long and short wavelength spin wave modes respectively.

In general, for $d=m$, the mean value of $S$ has a logarithmic divergence with system size, rather than a power law, which one might consider as a general characteristic of a critical system. It should be noted, however, that in the $2 D-X Y$ model the derivation sketched here relies on a low temperature approximation to the order parameter. The true order parameter has the same limit function $f(x)$, although it cannot be decomposed as a sum of statistically independent contributions: in that case the mean and all other moments of $S$ do diverge with system size as power laws [3]. The above results are therefore characteristic of a critical system. Indeed we believe that the frequent observation of $f(x)$ in nature is related the fact that the low temperature calculation, which is really the limit of criticality, captures the behaviour of a fully critical regime.

Putting $d=m=1$ in 4 gives the well-known Fisher-Tippet or Gumbel distribution, $G(x)$, one of the three limit functions that describes extreme value statistics [8, 9]. This result was recently derived by Antal et al and shown to correspond to the width fluctuations of a time series showing " $1 / \mathrm{f}$ noise" [10] and was confirmed on experimental data. In the past extremal statistics have often been used to model data from natural processes including river floods [5, 9]. The functions $f(x)$ and $G(x)$ are qualitatively quite similar, although $G(x)$ has a true exponential tail for fluctuations above the mean 8 while $f(x)$ has a pseudo exponential tail $\sim x \exp (-x)$. Despite this difference in the asymptotes, $f(x)$ is given to a good approximation over any physically oberservable range of fluctuations by $f(x) \sim G(x)^{a}$, where $a \approx \pi / 2[3, \pi$. We find that the fit of the Danube data to a generalized Gumbel function of this form is fairly insensitive to $a$, but that $f(x)$ gives a significantly better fit than $G(x)$. The precise relation of the universal fluctuation phenomena discussed here to 
extremal statistics remains is an interesting question that has been addressed in a very recent preprint by Dahlstedt and Jensen [11.

In conclusion, probability distributions for global quantities are generally rather hard to determine experimentally, limiting the usefulness of the prediction that these will often follow the form $f(x)$ [1]. However, in view of the fact that $f(x)$ describes the Danube data very well, it would appear reasonable to conclude that water level is, in this case, effectively a global measure of a complex system at steady state. In view of this result and that of [2], it would be interesting to test data for other rivers as well as appropriate financial data for further experimental evidence of the proposed far-reaching universality.

\section{REFERENCES}

[1] S. T. Bramwell, P. C. W. Holdsworth and J.-F. Pinton, Nature 396, 552 (1998).

[2] I. M. Jánosi and J. A. C. Gallas, Physica A 271, 448 (1999).

[3] S. T. Bramwell, J.-Y. Fortin, P. C. W. Holdsworth, S. Peysson, J.-F. Pinton, B. Portelli and M. Sellitto, Phys. Rev. E 6304 (4):1106 (2001).

[4] S. T. Bramwell, K. Christensen, J.-Y. Fortin, P. C. W. Holdsworth, H. J. Jensen, S. Lise, J. M. Lopez, M. Nicodemi, J.-F. Pinton and M. Sellitto, Phys. Rev. Lett. 84, 3744 (2000).

[5] See, for example, E. M Shaw, "Hydrology in Practice", 3rd ed., Chapman and Hall, London (1994).

[6] M. H. R. Stanley, L. A. N. Amaral, S. V. Buldyrev, S. Havlin, H. Leschhorn, P. Maass, M. A. Salinger and H. E. Stanley, Nature 379, 804 (1996).

[7] G. H. Wannier, "Statistical Physics", Dover (1987).

[8] A. Stuart and J. K. Ord., "Kendall's Advanced Theory of Statistics, Vol.1, Distribution Theory", London : Edward Arnold, 1994.

[9] E. J. Gumbel, "Statistics of Extremes", Columbia University Press, New York (1958).

[10] T. Antal, M. Droz, G. Gyorgyi and Z. Rácz, cond-mat/0105599.

[11] K. Dahlstedt and H. J. Jensen, cond-mat/0108007.

Acknowledgements: We thank I. M Jánosi for generously providing us with the Danube data. It is also a pleasure to thank L. Berthier, L. Bocquet, H. Jensen, J.-F. Pinton and Z. Rácz for stimulating discussions. 